\title{
Bevacizumab and Weekly Docetaxel in Patients with Metastatic Castrate-Resistant Prostate Cancer Previously Exposed to Docetaxel
}

\author{
Filippo Francini, ${ }^{1}$ Alessandra Pascucci, ${ }^{2}$ Edoardo Francini, ${ }^{2}$ Gianluca Bargagli, ${ }^{2}$ \\ Raffaele Conca, ${ }^{2}$ Antonella Licchetta, ${ }^{2}$ Giandomenico Roviello, ${ }^{2}$ Ignazio Martellucci, ${ }^{2}$ \\ Giorgio Chiriacò, ${ }^{2}$ Salvatora Tindara Miano, ${ }^{2}$ Giuseppe Marzocca, ${ }^{3}$ Antonio Manganelli, ${ }^{4}$ \\ Roberto Ponchietti, ${ }^{5}$ Vinno Savelli, ${ }^{6}$ and Roberto Petrioli ${ }^{2}$ \\ ${ }^{1}$ Department of Odontostomatology and Maxillo-Facial Surgery, University of Siena, Policlinico Le Scotte, \\ Viale Bracci 11, 53100 Siena, Italy \\ ${ }^{2}$ Medical Oncology, University of Siena, Policlinico Le Scotte, Viale Bracci 11, 53100 Siena, Italy \\ ${ }^{3}$ General Surgery, University of Siena, Policlinico Le Scotte, Viale Bracci 11, 53100 Siena, Italy \\ ${ }^{4}$ Urologic Surgery, University of Siena, Policlinico Le Scotte, Viale Bracci 11, 53100 Siena, Italy \\ ${ }^{5}$ Genitourinary Unit, University of Siena, Policlinico Le Scotte, Viale Bracci 11, 53100 Siena, Italy \\ ${ }^{6}$ Department of Surgery, University of Siena, Policlinico Le Scotte, Viale Bracci 11, 53100 Siena, Italy
}

Correspondence should be addressed to Roberto Petrioli, r.petrioli@ao-siena.toscana.it

Received 22 March 2011; Accepted 21 June 2011

Academic Editor: Ravi A. Madan

Copyright (C) 2011 Filippo Francini et al. This is an open access article distributed under the Creative Commons Attribution License, which permits unrestricted use, distribution, and reproduction in any medium, provided the original work is properly cited.

\begin{abstract}
Background. The aim of this paper was to evaluate the activity and tolerability of docetaxel (D) and bevacizumab (Bev) in patients with metastatic castrate-resistant prostate cancer (CRPC) previously exposed to D. Methods. Treatment consisted of D $30 \mathrm{mg} / \mathrm{m}^{2}$ i.v. for four consecutive weekly administrations followed by a 2-week rest interval, in addition to Bev $5 \mathrm{mg} / \mathrm{kg}$ i.v. every 2 weeks. Results. Forty-three patients were enrolled: a PSA response was observed in 27 patients (62.7\%, 95\% CI: 0.41 to 0.91$)$, and a palliative response was achieved in 31 patients (72.1\%, 95\%CI: 0.48 to 1.02 ). After a median followup of 11.3 months, only five patients had died. The regimen was generally well tolerated. Conclusion. Weekly D + biweekly Bev seems to be an effective and well-tolerated treatment option for patients with metastatic CRPC previously exposed to D-based chemotherapy.
\end{abstract}

\section{Introduction}

The results of two large randomised trials have provided substantial support in favor of the role of chemotherapy in the treatment of castrate-resistant prostate cancer (CRPC) by demonstrating that docetaxel $(\mathrm{D})$ and prednisone $(\mathrm{P})$ improve survival in comparison with older regimens and significantly improve the quality of life $[1,2]$. Therefore, $\mathrm{D}$ has become the first-line standard of care for metastatic CRPC, with PSA responses of about 50\% and median survivals of usually less than 20 months.

Patients with CRPC who progress after D treatment may be considered for a second-line chemotherapy, especially if they have a reasonable performance status, have symptoms, and/or are likely to soon develop symptoms for their disease. In this setting, a recent randomized phase III trial demonstrated that cabazitaxel, a tubuline-binding taxane drug, improved survival in metastatic CRPC patients with progressive disease after $\mathrm{D}$-treatment, with a $30 \%$ reduction in the risk of death compared with mitoxantrone taken as control group [3].

$\mathrm{D}$ resistance is a common problem in the treatment of many tumors including CRPC, and the development of new drugs that may overcome such resistance is important to extend D activity [4].

Angiogenesis is an important process for growth, progression, and metastasis of solid tumors, and the inhibitor of the vascular endothelial growth factor (VEGF) bevacizumab 
(Bev) is currently approved for the treatment of colon, lung, breast, and clear cell renal carcinoma in the metastatic setting [5].

In addition, preclinical data demonstrated that VEGF inhibition may also prevent further tumor growth of the prostate cancer cell line DU 145 implanted in nude mice, and preliminary clinical studies suggested that Bev combined with chemotherapy is tolerable and has promising activity in CRPC patients $[6,7]$.

Although Bev achieved no PSA response when it was used in monotherapy, interesting results were reported by the combination of Bev with D and estramustine as first-line treatment in a previous study of the Cancer and Leukemia Group B [8]. Moreover, a recent study described promising data in terms of PSA response and objective response in pretreated patients with CRPC receiving D and Bev [9]. On these previous experiences, and in the hypothesis that Bev may overcome the resistance to $\mathrm{D}$, we tested the activity and tolerability of Bev combined with D in CRPC patients with disease progression during or after D-based first-line chemotherapy.

\section{Patients and Methods}

2.1. Eligibility Criteria. This phase II study involved patients with histologically confirmed, measurable, or evaluable advanced prostatic adenocarcinoma who had progressed while on $\mathrm{D}$ or within 60 days after the last $\mathrm{D}$ dose. This last eligibility criterium, together with a minimum of 3 months of D-based chemotherapy as first-line treatment, was required in order to better elucidate the benefit of the addition of Bev. Patients were admitted to the chemotherapy protocol provided that they met at least one of the following criteria: a positive bone scan $a \geq 25 \%$ increase in PSA (PSA higher than $2 \mathrm{ng} / \mathrm{mL}$ ) in comparison with baseline on two successive occasions separated by at least two weeks for patients without measurable disease; new metastatic lesions revealed by a bone scan; and a $\geq 25 \%$ increase in a bidimensionally measurable tumor mass. All of the patients had to have an Eastern Cooperative Oncology Group (ECOG) performance status (PS) of $\leq 2$, adequate hematological (leukocytes $\geq 3000 / \mathrm{mm}^{3}$; hemoglobin $\geq 10 \mathrm{~g} / \mathrm{dL}$, platelets $\geq 100,000 / \mathrm{mm}^{3}$ ), renal (serum creatinine $\leq 2.0 \mathrm{mg} / \mathrm{dL}$ ), and hepatic function (serum bilirubin $\leq 2.0 \mathrm{mg} / \mathrm{dL}$; Table 1 ).

Patients were excluded if they had not received prior D-based chemotherapy or if they had congestive heart failure, a recent myocardial infarction, or any other previous malignant diseases except basal cell carcinoma of the skin. Bisphosphonates were admitted in all of patients who presented with bone metastases.

The study was approved by the ethical committee of Siena University, and all patients provided their written informed consent.

2.2. Treatment Plan. Treatment consisted of D $30 \mathrm{mg} / \mathrm{m}^{2}$ as a 30-minute intravenous infusion, using a schedule of four consecutive weekly administrations followed by a 2-week rest interval, in addition to Bev $5 \mathrm{mg} / \mathrm{kg}$ intravenously every 2
TABLE 1: Main eligibility criteria.

Histologically confirmed advanced prostatic carcinoma

Progression while on D or within 60 days after the last $\mathrm{D}$ dose

A positive bone scan and $a \geq 25 \%$ increase in PSA in comparison with baseline

New metastatic lesions revealed by a bone scan

A $\geq 25 \%$ increase in bidimensionally measurable tumor mass

ECOG PS of $\leq 2$

Leukocytes $\geq 3000 / \mathrm{mm}^{3}$, haemoglobin $\geq 10 \mathrm{~g} / \mathrm{dL}$, platelets

$\geq 100.000 / \mathrm{mm}^{3}$

Serum creatinine $\leq 2.0 \mathrm{mg} / \mathrm{dL}$; serum bilirubin $\leq 2.0 \mathrm{mg} / \mathrm{dL}$

weeks. Premedication consisted of P $10 \mathrm{mg}$ p.o. (12 h before, at the time of, and $12 \mathrm{~h}$ after $\mathrm{D}$ administration). Cycles were administered if serum leukocytes were $\geq 3000 / \mathrm{mm}^{3}$, granulocytes $>1500 / \mathrm{mm}^{3}$, and platelets $>100,000 / \mathrm{mm}^{3}$. Ondansetron $8 \mathrm{mg}$ was administered at the beginning of each treatment cycle as antiemetic medication. The patients continued to take analgesic medication at doses adjusted to provide optimal pain control. The chemotherapy was administered until disease progression or unacceptable toxicity, and for a maximum of 30 weekly $\mathrm{D}$ cycles. In responding patients, Bev could be continued at the investigator's discretion, or until disease progression or unacceptable toxicity.

2.3. Response Assessments. Tumor response in patients with measurable lesions was evaluated using the RECIST criteria [10]. Serum PSA was measured every three weeks: a PSA response was defined as a reduction from baseline of at least $50 \%$ for at least three weeks whereas PSA progression was defined as an increase from nadir of at least $25 \%$ and $\geq 2 \mathrm{ng} / \mathrm{mL}$ [11]. Pain symptomatology was measured at baseline and then every 6 weeks by the McGill Melzack Pain Questionnaire, and pain response was defined as a 2point reduction in the 6-point present pain intensity scale (or the complete disappearance of pain if the initial score was 1+) [12]. These results had to be maintained at two consecutive evaluations made at least 3 weeks apart and without any increase in analgesic consumption. The patients were asked to classify the average pain level during the previous $24 \mathrm{~h}$. We used a translated form of the McGill Melzack Questionnaire to which the "reconstruction-based methodology" has been applied [13]. Analgesic consumption was based on the average daily quantities taken by the patient during the previous week, and assigned oral morphine equivalents before analysis [14].

The laboratory studies (blood and platelet counts, and a comprehensive screening profile) were performed at baseline and every three weeks, and the patients underwent a weekly complete blood cell count and electrolytes profile before chemotherapy.

The imaging studies included abdominal and pelvic CT or magnetic resonance imaging, a bone scan, and chest radiography. All measurable diseases were reevaluated at 8week intervals. Radionuclide bone scans were repeated after 3 months. In all subjects, fasting venous blood samples were 
drawn between 8.00 and 9.00 a.m. after a 12 -h fasting period at baseline and after 3 months in order to assess the bone resorption marker crosslinked C-terminal telopeptide (CTX) and the bone formation marker bone alkaline phosphatase (B-ALP).

In all cases, a baseline ECG was obtained, and a further cardiac work-up was performed if indicated. Bone disease progression was defined as the appearance of any new bone lesion or the progression of existing bone metastases. A dental examination, including orthopantomography (OPT), was performed in all patients at baseline, and active dental surveillance every three months.

2.4. Treatment-Related Adverse Events. Toxicity was defined using the National Cancer Institute (NCI) Common Toxicity Criteria, version 3.0. The treatment was delayed at the first occurrence of grade II hematological toxicity, and administered at the same dose after it returned to grade I or better. In the case of grade III or IV toxicity, the treatment was interrupted and a maximum of three weeks was allowed for recovery, after which the patients were withdrawn from the study. In the case of a second episode of grade III or IV toxicity in the same patient, treatment was resumed after recovery and the subsequent administration of D was reduced to $20 \mathrm{mg} / \mathrm{m}^{2}$. Chemotherapy protocol was discontinued if the ejection fraction decreased below the institutional lower limit of normal and declined by $\geq 15 \%$.

2.5. Statistical Considerations. The primary endpoint was PSA response. In accordance with Simon's "optimal design", a sample size of 36 patients was planned, assuming a response rate of approximately $10 \%$ for other second-line chemotherapies, and a target level of interest of $30 \%$, with an $\alpha$ of 0.05 and a $\beta$ of 0.90 . In the hypothesis of $10 \%-$ $20 \%$ inevaluable patients, about 40 patients were planned to be enrolled to better estimate the response. Secondary endpoints were pain response, progression-free survival (PFS), and overall survival. PFS was defined as the time from starting chemotherapy to the first occurrence of objective or PSA progression, or death due to any cause.

\section{Results}

From September 2008 to April 2010, 43 patients were enrolled. Their median age was 74 years (range 58-82 years) Thirty-seven patients had bone metastases. and seventeen patients had measurable disease (Table 1). Most of enrolled patients have participated in a randomized phase II study which compared the combination of weekly D and weekly Epirubicin (EPI) with the conventional 3-weekly D [15]. All patients who had achieved a response or a stable disease during first-line chemotherapy had been retreated with $\mathrm{D}$ based chemotherapy. The median dose of $\mathrm{D}$ received before the enrollment in the current study was $940.8 \mathrm{mg} / \mathrm{m}^{2}$ (range 30-1122,3).

All enrolled patients were treated with the new treatment regimen within 60 days from the end of last $\mathrm{D}$ dose (range 12 to 52 days). Two patients received only one weekly
TABLE 2: Main patient characteristics.

\begin{tabular}{lc}
\hline Enrolled patients & 43 \\
\hline Median age (range): years & $74(58-82)$ \\
$\geq 75$ years & $21(48.8 \%)$ \\
\hline ECOG performance status & 9 \\
0 & 34 \\
\hline $1-2$ & 26 \\
\hline Sites of metastases & 4 \\
Bone & 4 \\
Bone + prostate cancer & 3 \\
Bone + prostate cancer + lymph nodes & 3 \\
Bone + lung & 2 \\
Prostate cancer + lymph nodes & 1 \\
Liver + lymph nodes & $78(47-374)$ \\
Prostate cancer + lung & 31 \\
\hline Median PSA (range), ng/mL & 9 \\
\hline Previous treatment & \\
Prostatectomy & 28 \\
Radiotherapy & 15 \\
\hline Hormone therapy & \\
1 & 21 \\
$\geq 2$ & 15 \\
\hline Prior first-line chemotherapy & 73 \\
w-Epirubicin + w-docetaxel & 23 \\
3-w Docetaxel + prednisone & \\
w-Docetaxel + prednisone & \\
\hline Number of chemotherapy regimens & \\
2 & \\
$>2$ & \\
\hline Prior third-line chemotherapy & \\
Docetaxel + prednisone & \\
\hline Bestepone & \\
\hline & \\
\hline
\end{tabular}

Best response to prior first-line

chemotherapy

PSA decline $\geq 50 \%$

Stable disease 7

Progressive disease 5

Baseline pain intensity

0

$1+8$

222

328

$4+5$

\begin{tabular}{lc}
5 & 0 \\
\hline Median hemoglobin, g/dL & 10.4 \\
Range & $7.9-13.8$
\end{tabular}

chemotherapy cycle for treatment-unrelated reasons. Two patients were lost to followup after four and six months from the start of treatment. All patients were included in the overall analysis (intent-to-treat). A total of 968 weekly D 
TABle 3: Response to treatment.

\begin{tabular}{lc}
\hline Enrolled patients & 43 \\
\hline Biochemical response & \\
PSA decline $\geq 50 \%$ & $27(62.8 \%)$ \\
Stable disease & $9(20.9 \%)$ \\
Progressive disease & $7(16.3 \%)$ \\
\hline Objective response & \\
Partial remission & $8 / 17(47.1 \%)$ \\
Stable disease & $7 / 17(41.1 \%)$ \\
Progressive disease & $2 / 17(11.8 \%)$ \\
\hline Palliative response & $31(72.1 \%)$ \\
\hline
\end{tabular}

TABle 4: Number of patients experiencing the most frequent treatment-related adverse events.

\begin{tabular}{lccc}
\hline & Grade 1 & Grade 2 & Grade 3 \\
\hline Hematological & & & \\
Neutropenia & $19(44.1 \%)$ & $14(32.5 \%)$ & $8(18.6 \%)$ \\
Anemia & $20(46.5 \%)$ & $15(34.8 \%)$ & $6(13.9 \%)$ \\
Thrombocytopenia & $18(41.8 \%)$ & $12(27.9 \%)$ & $4(9.3 \%)$ \\
\hline Nonhematological & & & \\
Nausea/vomiting & $12(27.9 \%)$ & $8(18.6 \%)$ & 0 \\
Diarrhea & $9(20.9 \%)$ & $6(13.9 \%)$ & 0 \\
Constipation & $13(30.2)$ & $11(25.5 \%)$ & 0 \\
Nail changes & $22(51.1 \%)$ & $17(39.5 \%)$ & $2(4.6 \%)$ \\
Dry eye/tearing & $26(60.4 \%)$ & $15(34.8 \%)$ & 0 \\
Myalgia/arthralgia & $22(51.1 \%)$ & $14(32.5 \%)$ & 0 \\
Fatigue & $21(48.8 \%)$ & $18(41.8 \%)$ & $2(4.6 \%)$ \\
Sensory neuropathy & $16(37.2 \%)$ & $7(16.2 \%)$ & 0 \\
Peripheral edema & $22(51.1 \%)$ & $8(18.6 \%)$ & 0 \\
Epistaxis & $23(53.4 \%)$ & $7(16.2 \%)$ & $1(2.3 \%)$ \\
Dyspnea & $12(27.9 \%)$ & $5(11.6 \%)$ & 0 \\
\hline
\end{tabular}

cycles (median 21, range 11-30) and a total of 1172 biweekly cycles of Bev (median 26, range 6-41) were administered.

3.1. Biochemical Response. A decrease in PSA levels $>50 \%$ was observed in 27 patients $(62.7 \%, 95 \%$ CI: 0.41 to 0.91$)$, and nine patients (20.9\%) had stable PSA for at least twelve weeks (Table 3 ). After the first 3 weekly cycles a PSA surge was observed in 18 out of 27 responding patients: in all these patients PSA then progressively decreased and at the third month was less than $50 \%$ with respect to the baseline values (Figure 1).

During the prior first-line chemotherapy, 15 out of the 27 responding patients had achieved PSA response while 8 had achieved stable disease and 4 patients had progressed.

3.2. Objective Response. Of seventeen patients with measurable disease, eight achieved PR and seven had stable disease: objective responses were observed on prostate cancer (3 cases), prostate cancer and pelvic lymph nodes (3 cases), and prostate cancer and lung metastases ( 2 cases).

The bone scan, which could be repeated after 3 months of treatment in 35 out of 37 patients with bone metastases,

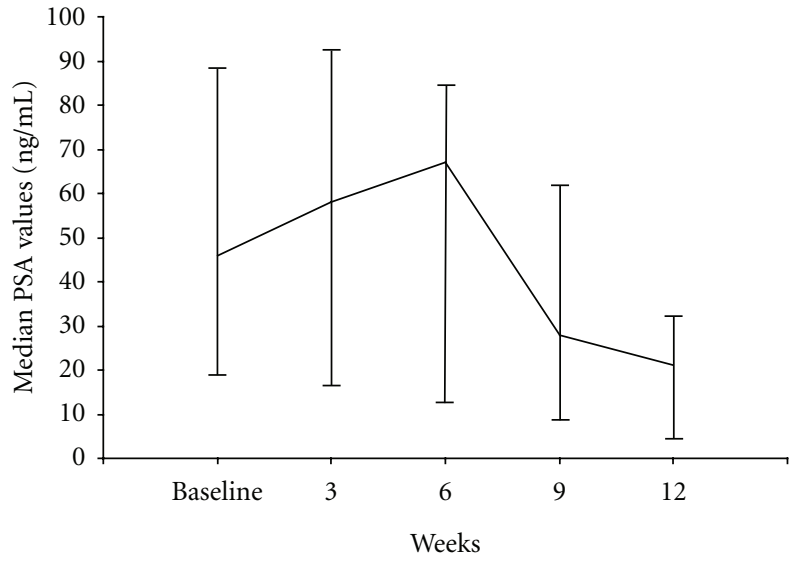

FIgURE 1: Median PSA (with minimum and maximum values) in 18 out of 27 responding patients who showed a PSA surge within the first 3 months of treatment with weekly D + biweekly Bev.

showed stable disease in 29 patients, and partial remission in 5 patients; two or more new lesions compared with the prior scan for trial entry were described in one patient. This same patient had PSA progression after 3 months and chemotherapy was stopped. The bone markers CTX and BALP were reduced $>50 \%$ with respect to baseline values in 33 and 28 patients, respectively, after 12 weeks from the start of treatment (65\% median reduction for CTX and 58\% median reduction for B-ALP). A palliative response was observed in 31 patients after 12 weeks ( $72.1 \%, 95 \%$ CI: to 0.48 to 1.02$)$.

After 12 weeks from the start of the new treatment protocol, seven patients (including two subjects who received only one treatment cycle) had PSA progression, but three of them had a reduction in bone pain with decrease in analgesics use, improvement in performance status, and reduction in serum levels of the bone markers CTX and BALP. Despite the initial end-point of the study, because of the achieved clinical benefit, our oncology group and the scientific ethical committee decided to continue $\mathrm{D}+$ $\mathrm{Bev}$ in these three patients until worsening of pain and/or performance status.

After a median followup of 11.3 months, 18 patients showed PSA progression and only five patients had died.

The regimen was generally well tolerated, and no unexpected toxic effects were observed (Table 4). No grade 4 toxicity or congestive heart failure was observed, and all cycles were administered on an outpatient basis. The most frequent side effects were neutropenia, anemia, thrombocytopenia, epistaxis, and fatigue, which were grade I or II in most cases. Grade III fatigue was observed in two patients after nine and sixteen cycles, respectively: despite the reduction of $\mathrm{D}$ dose and the discontinuation of Bev, treatment was then interrupted in these cases because of the persistence of this side effect. Grade 1 epistaxis was observed in 23 patients $(53.4 \%)$ during treatment, but reached grade 2 in only 7 cases and grade 3 in one patient. No patient developed osteonecrosis of the jaw (ONJ). Dose reduction of D was required in a total of twelve patients: $37(3.8 \%)$ weekly D 
cycles were administered with a $33 \%$ reduction, down to $20 \mathrm{mg}$ weekly. A total of 57 (5.8\%) weekly D cycles were delayed: the reason for the delays were hematological in 41 (71.9\%) and nonhematological in $16(28.1 \%)$ cycles. A total of 46 (39.2\%) biweekly Bev cycles were delayed: the reasons for the delays were haematological in 39 (84.8\%) and nonhematological in 7 (15.2\%) cycles.

\section{Discussion}

The currents phase II study is the first extended report which suggests that the combination of weekly D with the biweekly administration of the antiangiogenic agent Bev is effective and tolerable in the treatment of patients with metastatic CRPC who have progressed after D-based chemotherapy: the $62.7 \%$ PSA and $72.1 \%$ palliative response compare favorably with the results observed in phase II studies of secondline chemotherapy $[16,17]$. Other chemotherapeutic agents might be used after initial treatment with $\mathrm{D}$, including old drugs such as vinorelbine, oral cyclophosphamide, etoposide, mitoxantrone, vinblastine, and doxorubicin, but most studies reported no objective response and less than 15\% laboratory response [18]. Other studies described modest activity with satraplatin, a third-generation platinum analog, or ixabepilone, an epothilone, with mitoxantrone, or also with the combination of D and high-dose calcitriol [19-21].

It must be remembered that in most clinical trials a few patients stop the first-line treatment with $\mathrm{D}$ while still responding to the drug. In this group of patients a repeated treatment with $\mathrm{D}$ might be appropriated if progression occurs after a reasonably long time interval [22]. In our study, the strict eligibility requirement of progression while on $\mathrm{D}$ or within 60 days of the last $\mathrm{D}$ dose means that these patients might have not responded to rechallenge with D. Notably, responses were seen also in patients who had not shown an initial response to prior D as first-line treatment. Therefore, this finding and the characteristics of enrolled patients support an effective role of Bev in restoring the sensitivity to $\mathrm{D}$ and also in reversing resistance in patients who were previously nonresponders to the drug (Table 1). Bev is able to alter tumor vasculature, for example, decreasing tumor vessel permeability and increasing intratumoral perfusion, which might turn into an improved tumor delivery of a cytotoxic agent, thus enhancing its antitumor activity [23]. Since the limited tissue penetration is an important mechanism of tumor resistance to taxanes, the effects of the antiangiogenic agent Bev may be a possible explanation of the observed reversal of $\mathrm{D}$ resistance in our population study [24].

Another point to consider is the observed PSA surges during treatment protocol in 18 out of 27 responding patients, which may suggest massive cancer cell death and PSA release, thus indicating efficacy, as also reported in other previous trials during chemotherapy for CRPC $[25,26]$. Nevertheless, despite the unknown biological relevance of this transient initial PSA increase, most of our patients achieved an improvement in bone symptomatology and in performance status, and PSA decreased $>50 \%$ at 3 months
(Figure 1). Furthermore, PSA results correlated with changes in bone markers, since CTX and B-ALP were reduced with respect to baseline values in all these patients, and this was probably related to the real antitumor activity of the $\mathrm{D}+\mathrm{Bev}$ combination.

Notably, despite PSA progression after 3 months of treatment, three patients continued to have an improvement in performance status and reduction in bone symptomatology and bone markers, and because of this achieved clinical benefit they continued to receive $\mathrm{D}+\mathrm{Bev}$. This finding may confirm that the progression criteria that are usually suitable for assessment of efficacy of cytotoxic agents in CRPC may not be suitable for discriminating treatment effects of targeted agents such as Bev [27, 28]. It may be that significant treatment benefits with targeted therapies need long time scales to emerge, possibly due to its noncytotoxictargeted mechanism of action.

As Bev-based salvage treatment, a 55\% PSA response and $37.5 \%$ objective response was found in 20 pretreated patients with CRPC receiving D $60 \mathrm{mg} / \mathrm{m}^{2}$ and Bev $10 \mathrm{mg} / \mathrm{kg}$ every 3 weeks [9]. Another recent experience suggested a benefit in progression-free and in overall survival by the use of weekly D $25 \mathrm{mg} / \mathrm{m}^{2}$ combined with Bev $10 \mathrm{mg} / \mathrm{kg}$ every two weeks in CRPC patients [29]. The toxicity profile of our treatment protocol was comparable to that observed in these BEV-based salvage treatments, with a major incidence of grade IV neutropenia and thrombocytopenia reported by the use of 3-weekly D schedule. Considering the strict eligibility criteria of our study and the fact that all our patients had previously received at least two chemotherapy lines, the current results appear even more encouraging than that found in the aforementioned reports. Nevertheless, it must be considered that most of metastatic CRPC patients who relapse after the first line $\mathrm{D}$ and $\mathrm{D}$ rechallenge do not survive more than 6 months. It is notable that in the current study, after a median follow-up time of 11.3 months, only five patients had died and most patients who started the new treatment protocol more that 12 months ago are still alive and have a good quality of life.

Therefore, although three-weekly $\mathrm{D}$ and $\mathrm{P}$ remains the conventional treatment protocol in first-line setting, it may be hypothesized that weekly D combined with biweekly Bev, as applied by us, is an appropriate schedule in terms of activity and toxicity for heavily pretreated patients. The combination of weekly scheduling of D with Bev has shown interesting activity without significant toxicity also in breast, ovarian and mesenchymal tumors [30-32].

The efficacy and safety results of the current study compare favorably also with those reported with the drug cabazitaxel, that was recently approved by US Food and Drug administration for second-line treatment of metastatic CRPC patients [3]. Severe neutropenia was common in cabazitaxel trial $(89 \%)$, and $18 \%$ of patients discontinued the study treatment because of adverse events while grade III neutropenia was observed in only $18.6 \%$ of cases in our population study. Nevertheless toxicity was mild in our patients: adverse events likely related to Bev (hypertension, epistaxis, and albuminuria) never reached grade 3 and were easily manageable, as usually reported in other tumors with 
the biweekly schedule of $5 \mathrm{mg} / \mathrm{kg}$ of Bev. The low toxicity of $\mathrm{D}$ was mainly due to the weekly schedule. The efficacy of weekly D seems to be similar to that of the usual 3-weekly schedule, but their comparative toxicities differ markedly, with moderate to severe myelosuppression being common when the drug is administered once every 3 weeks [33]. As well as is concerned other adverse events, Altough the new and potent antiangiogenic therapies might theoretically enhance the antiangiogenic effects of zoledronic acid on bone tissue, our findings do not suggest a trend for a possible higher incidence of bisphosphonate-induced ONJ for patients receiving zoledronic acid and Bev [34].

Another point to consider is that the percentage of enrolled patients $\geq 75$ years was about $50 \%$ in the current study, compared with only $18 \%$ in cabazitaxel trial. On these findings, it seems that weekly D and biweekly Bev can be safety administered also to elderly patients, who represent the most part of CRPC population. Therefore, although cabazitaxel will be the only established second-line treatment of CRPC patients in the next future, weekly D and Bev may be a valid option for patients with a decreased hematological reserve and/or for elderly subjects. Moreover, in the absence of a randomized comparison between cabazitaxel and our proposed treatment protocol, weekly $\mathrm{D}$ and Bev might be used after cabazitaxel failure.

In conclusion the results of this study suggest that weekly $\mathrm{D}$ and biweekly Bev is an effective and well-tolerated treatment option for patients with metastatic CRPC previously exposed to D. Bev seems to overcome the resistance to the drug in patients who had progressed during or after D-based chemotherapy.

\section{Conclusion}

Weekly D + biweekly Bev seems to be an effective and well-tolerated treatment option for patients with metastatic CRPC previously exposed to D-based chemotherapy.

\section{Acknowledgments}

The authors wish to thank the following members of the Oncology Group on Genitourinary Tumors who substantially contributed to recruite eligible patients and made this study possible: Prof. F. Roviello, Prof. D. Marrelli, Oncologic Surgery, University of Siena; Dr. Massoni, Urology, Siena; Dr. G. Giusti, USL 10, Pisa, Italy; Dr. G. Vegni, Volterra Hospital; Dr. R. Terreni, Castelfiorentino, AUSL 11 Empoli, Dr. ssa T. Cerri Vestri, USL Arezzo.

\section{References}

[1] I. F. Tannock, R. de Wit, W. R. Berry et al., "Docetaxel plus prednisone or mitoxantrone plus prednisone for advanced prostate cancer," The New England Journal of Medicine, vol. 351, no. 15, pp. 1502-1512, 2004.

[2] D. P. Petrylak, C. M. Tangen, M. H. Hussain et al., "Docetaxel and estramustine compared with mitoxantrone and prednisone for advanced refractory prostate cancer," The New England Journal of Medicine, vol. 351, no. 15, pp. 1513-1520, 2004.
[3] J. S. de Bono, S. Oudard, M. Ozguroglu et al., "Prednisone plus cabazitaxel or mitoxantrone for metastatic castrationresistant prostate cancer progressing after docetaxel treatment: a randomised open-label trial," The Lancet, vol. 376, no. 9747, pp. 1147-1154, 2010.

[4] P. Mathew and R. DiPaola, "Taxane refractory prostate cancer," Journal of Urology, vol. 178, no. 3, part 2, pp. S36-S41, 2007.

[5] R. L. Pañares and A. A. Garcia, "Bevacizumab in the management of solid tumors," Expert Review of Anticancer Therapy, vol. 7, no. 4, pp. 433-445, 2007.

[6] P. Borgström, M. A. Bourdon, K. J. Hillan, P. Sriramarao, and N. Ferrara, "Neutralizing anti-vascular endothelial growth factor antibody completely inhibits angiogenesis and growth of human prostate carcinoma micro tumors in vivo," Prostate, vol. 35, no. 1, pp. 1-10, 1998.

[7] C. J. Ryan, A. M. Lin, and E. J. Small, "Angiogenesis inhibition plus chemotherapy for metastatic hormone refractory prostate cancer: history and rationale," Urologic Oncology, vol. 24, no. 3, pp. 250-253, 2006.

[8] J. Picus, S. Halabi, B. Rini et al., "The use of bevacizumab (B) with docetaxel (D) and estramustine (E) in hormone refractory prostate cancer (HRPC): initial results of CALGB 90006," Proceeding of the American Society of Clinical Oncology, vol. 22, abstract 1578, 2003.

[9] G. Di Lorenzo, W. D. Figg, S. D. Fossa et al., "Combination of bevacizumab and docetaxel in docetaxel-pretreated hormonerefractory prostate cancer: a phase 2 study," The European Urology, vol. 54, no. 5, pp. 1089-1096, 2008.

[10] P. Therasse, S. G. Arbuck, E. A. Eisenhauer et al., "New guidelines to evaluate the response to treatment in solid tumors," Journal of the National Cancer Institute, vol. 92, no. 3, pp. 205-216, 2000.

[11] H. I. Scher, S. Halabi, I. F. Tannock et al., "Design and end points of clinical trials for patients with progressive prostate cancer and castrate levels of testosterone: recommendations of the Prostate Cancer Clinical Trials Working Group," Journal of Clinical Oncology, vol. 26, no. 7, pp. 1148-1159, 2008.

[12] R. Melzack, "The McGill pain questionnaire: major properties and scoring methods," Pain, vol. 1, no. 3, pp. 277-299, 1975.

[13] G. De Benedittis, R. Massei, R. Nobili, and A. Pieri, "The Italian pain questionnaire," Pain, vol. 33, no. 1, pp. 53-62, 1988.

[14] A. McCormack, D. Hunter-Smith, Z. H. Piotrowski, M. Grant, S. Kubik, and K. Kessel, "Analgesic use in home hospice cancer patients," Journal of Family Practice, vol. 34, no. 2, pp. 160-164, 1992.

[15] R. Petrioli, A. Pascucci, R. Conca et al., "Docetaxel and epirubicin compared with docetaxel and prednisone in advanced castrate-resistant prostate cancer: a randomised phase II study," The British Journal of Cancer, vol. 104, no. 4, pp. 613619, 2011.

[16] D. R. Berthold, C. N. Sternberg, and I. F. Tannock, "Management of advanced prostate cancer after first-line chemotherapy," Journal of Clinical Oncology, vol. 23, no. 32, pp. 82478252, 2005.

[17] W. K. Oh, J. Manola, V. Babcic, N. Harnam, and P. W. Kantoff, "Response to second-line chemotherapy in patients with hormone refractory prostate cancer receiving two sequences of mitoxantrone and taxanes," Urology, vol. 67, no. 6, pp. 12351240, 2006.

[18] E. G. Garmey, O. Sartor, S. Halabi, and N. J. Vogelzang, "Second-line chemotherapy for advanced hormone-refractory prostate cancer," Clinical Advances in Hematology and Oncology, vol. 6, no. 2, pp. 118-122, 127-132, 2008. 
[19] C. N. Sternberg, "Satraplatin in the treatment of hormonerefractory prostate cancer," The British Journal of Urology International, vol. 96, no. 7, pp. 990-994, 2005.

[20] M. D. Galsky, E. J. Small, W. K. Oh et al., "Multi-institutional randomized phase II trial of the epothilone B analog ixabepilone (BMS-247550) with or without estramustine phosphate in patients with progressive castrate metastatic prostate cancer," Journal of Clinical Oncology, vol. 23, no. 7, pp. 1439-1446, 2005.

[21] R. Petrioli, A. Pascucci, E. Francini et al., "Weekly high-dose calcitriol and docetaxel in patients with metastatic hormonerefractory prostate cancer previously exposed to docetaxel," The British Journal of Urology International, vol. 100, no. 4, pp. 775-779, 2007.

[22] Y. Loriot, C. Massard, M. Gross-Goupil et al., "The interval from the last cycle of docetaxel-based chemotherapy to progression is associated with the efficacy of subsequent docetaxel in patients with prostate cancer," The European Journal of Cancer, vol. 46, no. 10, pp. 1770-1772, 2010.

[23] P. V. Dickson, J. B. Hamner, T. L. Sims et al., "Bevacizumabinduced transient remodeling of the vasculature in neuroblastoma xenografts results in improved delivery and efficacy of systemically administered chemotherapy," Clinical Cancer Research, vol. 13, no. 13, pp. 3942-3950, 2007.

[24] A. H. Kyle, L. A. Huxham, D. M. Yeoman, and A. I. Minchinton, "Limited tissue penetration of taxanes: a mechanism for resistance in solid tumors," Clinical Cancer Research, vol. 13, no. 9, pp. 2804-2810, 2007.

[25] R. Thuret, C. Massard, M. Gross-Goupil et al., "The postchemotherapy PSA surge syndrome," Annals of Oncology, vol. 19, no. 7, pp. 1308-1311, 2008.

[26] T. Nelius and S. Filleur, "PSA surge/flare-up in patients with castration-refractory prostate cancer during the initial phase of chemotherapy," Prostate, vol. 69, no. 16, pp. 1802-1807, 2009.

[27] L. Collette, T. Burzykowski, K. J. Carroll, D. Newling, T. Morris, and F. H. Schröder, "Is prostate-specific antigen a valid surrogate end point for survival in hormonally treated patients with metastatic prostate cancer? Joint research of the European Organisation for Research and treatment of cancer, the Limburgs Universitair Centrum, and AstraZeneca Pharmaceuticals," Journal of Clinical Oncology, vol. 23, no. 25, pp. 6139-6148, 2005.

[28] H. I. Scher, M. Warren, and G. Heller, "The association between measures of progression and survival in castratemetastatic prostate cancer," Clinical Cancer Research, vol. 13, no. 5, pp. 1488-1492, 2007.

[29] A. Heidenreich, D. A. Pfister, R. Thüer, and B. Brehmer, "Docetaxel rechallenge versus docetaxel/bevacizumab in castrationresistant prostate cancer following first-line docetaxel," Journal of Clinical Oncology, vol. 28, no. 15_supplement, abstract e15006, 2010.

[30] B. Ramaswamy, A. D. Elias, N. T. Kelbick et al., "Phase II trial of bevacizumab in combination with weekly docetaxel in metastatic breast cancer patients," Clinical Cancer Research, vol. 12, no. 10, pp. 3124-3129, 2006.

[31] D. E. Cohn, S. Valmadre, K. E. Resnick, L. A. Eaton, L. J. Copeland, and J. M. Fowler, "Bevacizumab and weekly taxane chemotherapy demonstrates activity in refractory ovarian cancer," Gynecologic Oncology, vol. 102, no. 2, pp. 134-139, 2006.

[32] B. Gorelik, I. Ziv, R. Shohat et al., "Efficacy of weekly docetaxel and bevacizumab in mesenchymal chondrosarcoma: a new theranostic method combining xenografted biopsies with a mathematical model," Cancer Research, vol. 68, no. 21, pp. 9033-9040, 2008.

[33] F. K. Engels and J. Verweij, "Docetaxel administration schedule: from fever to tears? A review of randomised studies," The European Journal of Cancer, vol. 41, no. 8, pp. 1117-1126, 2005.

[34] G. Ferretti, A. Fabi, P. Carlini et al., "Zoledronic acid and angiogenesis," Clinical Cancer Research, vol. 13, no. 22, part 1, p. $6850,2007$. 


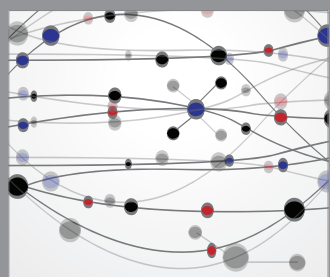

The Scientific World Journal
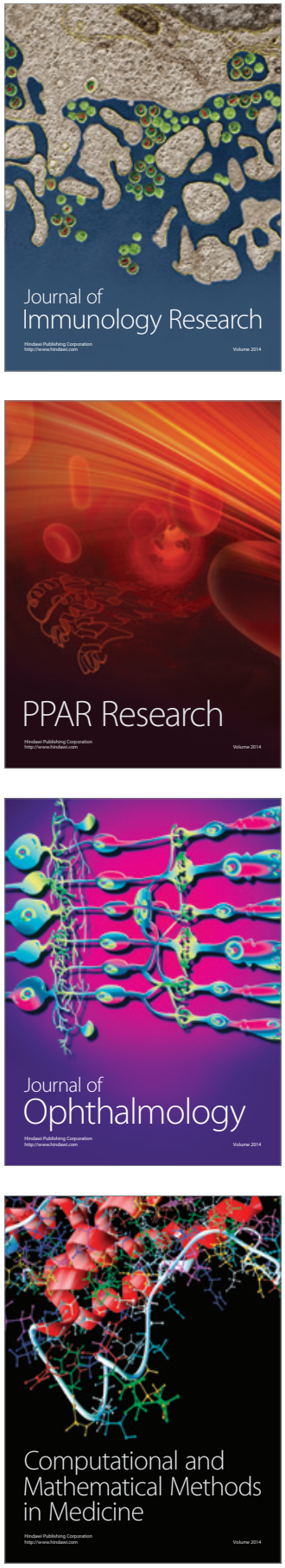

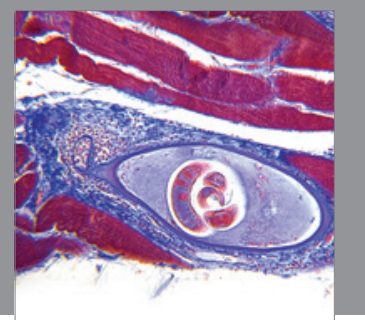

Gastroenterology

Research and Practice
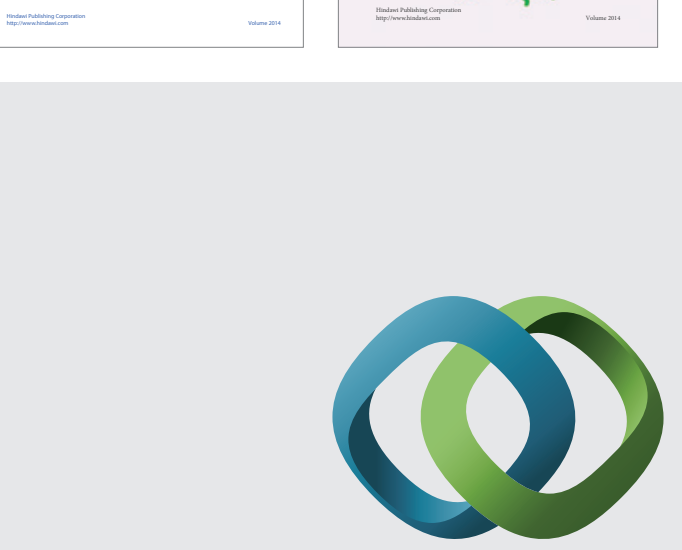

\section{Hindawi}

Submit your manuscripts at

http://www.hindawi.com
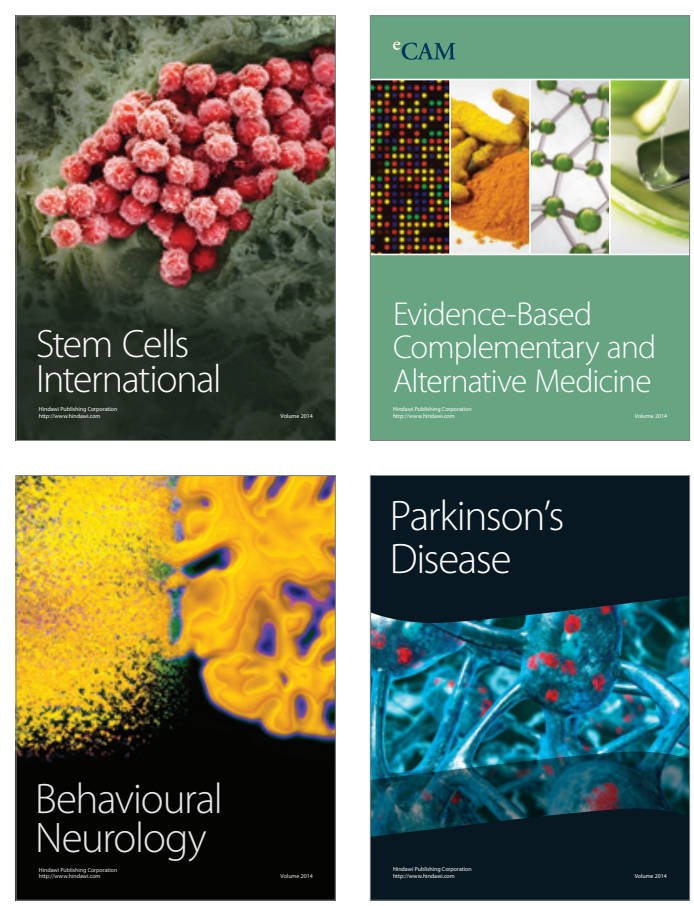

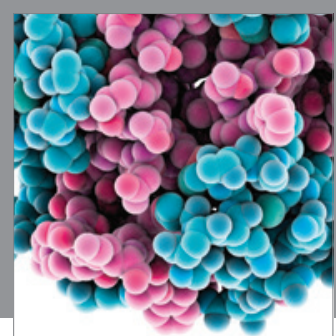

Journal of
Diabetes Research

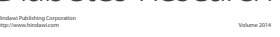

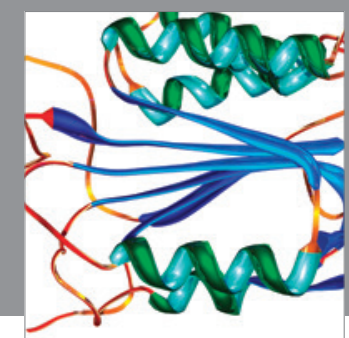

Disease Markers
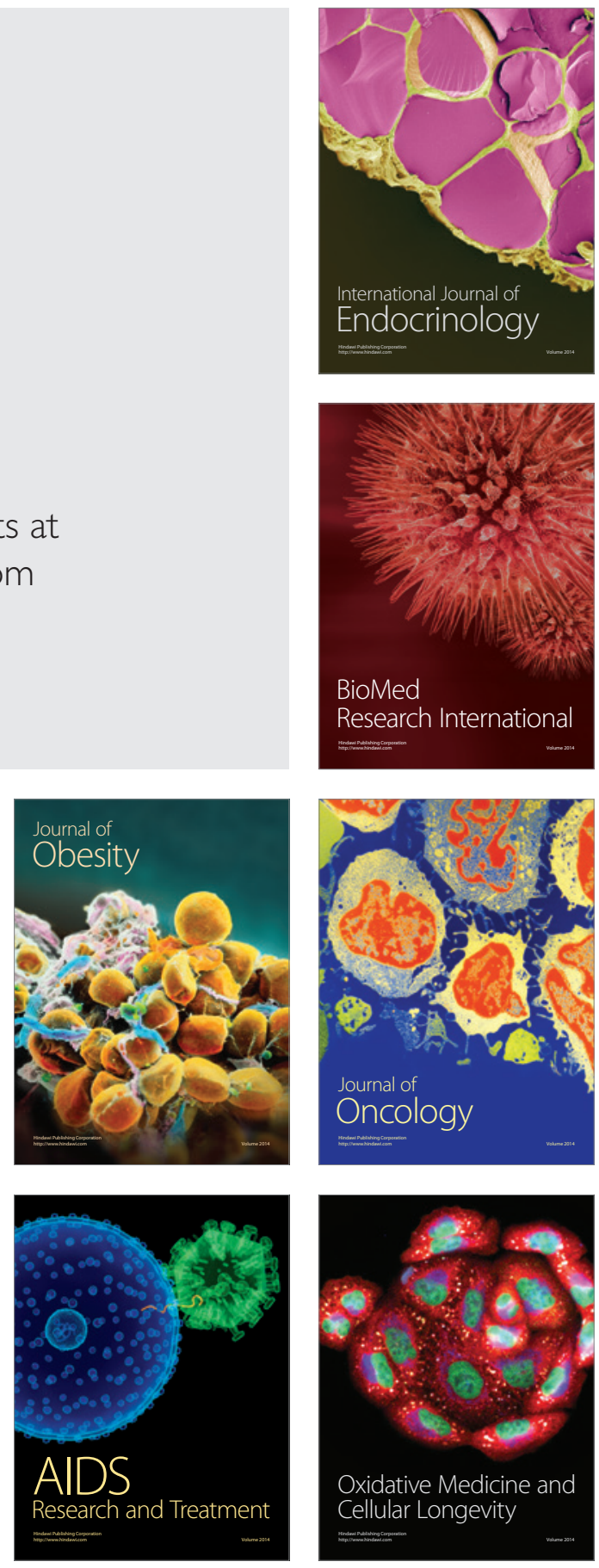